\title{
Holographic entanglement entropy for 4D conformal gravity
}

\author{
Mohsen Alishahiha, ${ }^{a}$ Amin Faraji Astaneh ${ }^{b, c}$ and M. Reza Mohammadi Mozaffar ${ }^{a}$ \\ ${ }^{a}$ School of Physics, Institute for Research in Fundamental Sciences (IPM), \\ P.O. Box 19395-5531, Tehran, Iran \\ ${ }^{b}$ Department of Physics, Sharif University of Technology, \\ P.O. Box 11365-9161, Tehran, Iran \\ ${ }^{c}$ School of Particles and Accelerators \\ Institute for Research in Fundamental Sciences (IPM), \\ P.O. Box 19395-5531, Tehran, Iran \\ E-mail: alishah@ipm.ir, faraji@ipm.ir, m_mohammadi@ipm.ir
}

ABSTRACT: Using the proposal for holographic entanglement entropy in higher derivative gravities, we compute holographic entanglement entropy for the conformal gravity in four dimensions which turns out to be finite. However, if one subtracts the contribution of the four dimensional Gauss-Bonnet term, the corresponding entanglement entropy has a divergent term and indeed restricted to an Einstein solution of the conformal gravity, the resultant entanglement entropy is exactly the same as that in the Einstein gravity. We will also make a comment on the first law of the entanglement thermodynamics for the conformal gravity in four dimensions.

KEYwords: Gauge-gravity correspondence, AdS-CFT Correspondence, Classical Theories of Gravity

ArXiv EPRINT: 1311.4329 


\section{Contents}

1 Introduction 1

2 Entanglement entropy for black brane solutions 4

2.1 Strip entangling region 4

2.2 Disk entangling region 9

3 Entanglement entropy for wave solution $\quad 12$

4 Discussions 14

\section{Introduction}

Four dimensional conformal gravity whose action is given in terms of the Weyl tensor, is a theory that is invariant under a Weyl transformation of the metric. This, indeed, leads to a theory which depends on angles, but not on distances. Unlike the Einstein gravity ${ }^{1}$ which is ghost free, the conformal gravity has ghost, though it is renormalizable $[1,2]$. It is also known that the solutions of Einstein gravity are solutions of the conformal gravity too, though the inverse is not necessarily correct. It is, however, possible to impose a certain boundary condition on the metric in the conformal gravity, so that the theory admits only Einstein solutions [3].

An interesting feature of the four dimensional conformal gravity is its relation to the Einstein gravity in four dimensions. Actually, it was shown [4] that the renormalized on shell action of a four dimensional Einstein gravity in an asymptotically hyperbolic Einstein spaces is given by the action of conformal gravity. Of course the action must be evaluated on an Einstein solution. Moreover the author of [3] has also argued that the certain boundary condition, mentioned above, removes the ghost from the theory and indeed the physical content of both theories would be the same.

Motivated by these observations, in the present paper, we will study the holographic entanglement entropy in the four dimensional conformal gravity with the aim of comparing the results with that of the Einstein gravity.

The action of the conformal gravity in four dimensions is

$$
\begin{aligned}
S & =-\frac{\kappa}{32 \pi} \int d^{4} x \sqrt{-g} C_{\mu \nu \rho \sigma} C^{\mu \nu \rho \sigma} \\
& =-\frac{\kappa}{32 \pi} \int d^{4} x \sqrt{-g}\left(R_{\mu \nu \rho \sigma} R^{\mu \nu \rho \sigma}-2 R_{\mu \nu} R^{\mu \nu}+\frac{1}{3} R^{2}\right) .
\end{aligned}
$$

\footnotetext{
${ }^{1}$ In this paper by "Einstein gravity" we mean an Einstein gravity with a cosmological constant.
} 
Here $\kappa$ is a dimensionless coupling constant which is the only free parameter of the theory. The corresponding equations of motion, which is essentially the vanishing of the Bach tensor, are

$$
\left(\nabla^{\mu} \nabla^{\nu}-\frac{1}{2} R^{\mu \nu}\right) C_{\mu \rho \sigma \nu}=0
$$

These equations admit black hole solutions as follows [5] (see also [6])

$$
d s^{2}=-F(r) d t^{2}+\frac{d r^{2}}{F(r)}+r^{2} d \Omega_{2}^{2}, \quad F(r)= \pm \sqrt{1+a m}-\frac{m}{r}-\frac{r^{2}}{L^{2}}+\frac{a r}{3},
$$

where $L$ is the radius of curvature and the \pm signs refer to two branches of the solutions [3]. In what follows we will only consider the plus sign, where the solutions are asymptotically AdS. It is also possible to consider the large volume limit [7] so that the resultant solutions will be black branes

$$
d s^{2}=\frac{L^{2}}{r^{2}}\left(-b(r) d t^{2}+\frac{d r^{2}}{b(r)}+\sum_{i=1}^{2} d x_{i}^{2}\right), \quad b(r)=1-\frac{a}{3} r \pm \sqrt{m a} r^{2}-m r^{3} .
$$

Here $a$ and $m$ are the parameters of the solutions. Note that if one sets $a=0$ in the above solutions, they reduce to AdS black hole (brane) solutions of the Einstein gravity in four dimensions.

On top of these solutions, we found another solution which is, indeed, an AdS wave solution (see also [8-10])

$$
d s^{2}=\frac{L^{2}}{r^{2}}\left(d r^{2}+d y^{2}-2 d x_{-} d x_{+}+k\left(x_{+}, r\right) d x_{+}^{2}\right)
$$

with

$$
k\left(x_{+}, r\right)=c_{0}\left(x_{+}\right)+c_{1}\left(x_{+}\right) r+c_{2}\left(x_{+}\right) r^{2}+c_{3}\left(x_{+}\right) r^{3} .
$$

For $c_{1}=c_{2}=0$ this solution reduces to an AdS wave solution in the Einstein gravity. Note that since the Weyl transformation is a symmetry of the model, rescaling the above solutions with an arbitrary function leads to new metrics which are still solutions of the model.

As we already mentioned the above solutions are not necessarily solutions of the Einstein gravity, though if one sets some of their parameters to zero they reduce to solutions of the Einstein gravity. Therefore it is natural to pose a question whether there is a systematic way one could remove these terms from the solutions. Actually the answer is yes. To explore the situation note that generally, using the Fefferman-Graham coordinates for a conformally asymptotically locally AdS solution, the equations of motion allow the following form for the metric

$$
d s^{2}=e^{\phi(r)} \frac{L^{2}}{r^{2}}\left(d r^{2}+g_{i j}(x, r) d x^{i} d x^{j}\right), \quad g_{i j}(x, r)=g_{i j}^{(0)}+g_{i j}^{(1)} r+g_{i j}^{(2)} r^{2}+g_{i j}^{(3)} r^{3}+\cdots
$$

It is then clear that by imposing a Neumann boundary condition as $\partial_{r} g_{i j} \mid=g_{i j}^{(1)}=0$ at the boundary one can remove the linear term leading to an Einstein solution [3]. This is 
indeed the ghost mode which is removed by the boundary condition. It is clear that this condition sets $a=0 .^{2}$

It is worth noting that in the context of holographic renormalization it was argued that this extra term would correspond to a source of a relevant operator in the dual conformal field theory $[11,12]$. More precisely, having non-zero linear term corresponds to deforming the corresponding dual theory with a relevant operator.

This is the aim of the present paper to compute the entanglement entropy of a three dimensional field theory whose gravitational dual is given by the four dimensional conformal gravity (1.1). Since the action of the conformal gravity in four dimensions contains higher derivative terms, the simple procedure of calculating the holographic entanglement entropy in terms of a minimal surface in the bulk [13] is not applicable. Therefore to compute the holographic entanglement entropy one should proceed with another method.

Actually using a method based on the regularization of squashed cones the authors of [14] proposed an expression for the holographic entanglement entropy for actions with curvature squared higher derivative terms. This proposal has been further studied in [15, 16] for certain higher derivative gravities. A general formula for the holographic entanglement entropy for higher derivative gravities has been also proposed in [17, 18]. In what follows we will use the procedure of [14] in which the corresponding entropy functional for our case becomes

$$
S_{A}=\frac{\kappa}{8} \int d^{2} \zeta \sqrt{h}\left[\frac{2}{3} R-2\left(R_{\mu \nu} n_{i}^{\mu} n_{i}^{\nu}-\frac{1}{2} \mathcal{K}^{i} \mathcal{K}_{i}\right)+2\left(R_{\mu \nu \rho \sigma} n_{i}^{\mu} n_{j}^{\nu} n_{i}^{\rho} n_{j}^{\sigma}-\mathcal{K}_{\mu \nu}^{i} \mathcal{K}_{i}^{\mu \nu}\right)\right]
$$

where $i=1,2$ denotes two transverse directions to a co-dimension two hypersurface in the bulk, $n_{i}^{\mu}$ are two unit mutually orthogonal normal vectors on the co-dimension two hypersurface and $\mathcal{K}^{(i)}$ is the trace of two extrinsic curvature tensors defined by

$$
\mathcal{K}_{\mu \nu}^{(i)}=\pi_{\mu}^{\sigma} \pi_{\nu}^{\rho} \nabla_{\rho}\left(n_{i}\right)_{\sigma}, \quad \text { with } \quad \pi_{\mu}^{\sigma}=\epsilon_{\mu}^{\sigma}+\xi \sum_{i=1,2}\left(n_{i}\right)^{\sigma}\left(n_{i}\right)_{\mu},
$$

where $\xi=-1$ for space-like and $\xi=1$ for time-like vectors. Moreover $h$ is the induced metric on the hypersurface whose coordinates are denoted by $\zeta$.

Now the procedure is to consider an entangling region on the dual field theory on the boundary, then consider a co-dimension two hypersurface in the bulk whose intersection with the boundary coincides with the boundary of the entangling region. The profile of the co-dimension two hypersurface may be obtained by minimizing the above entropy functional. Moreover the entanglement entropy is the value of the entropy functional evaluated on the co-dimension two hypersurface.

The main results of our paper are as follows. We found that the entanglement entropy of theories whose gravitational dual are provided by the four dimensional conformal gravity

\footnotetext{
${ }^{2}$ For the wave solution (1.5) in order to get an Einstein solution one needs to set both $c_{1}$ and $c_{2}$ to zero. But imposing the Neumann boundary condition leads to $c_{1}=0$ and $c_{2}$ could still be non-zero. More probably there should be another constrain on the solution to set either a relation between $c_{1}$ and $c_{2}$ or $c_{2}=0$, though we could not realize it. We should admit the resolution is not clear to us. Nevertheless since we are just interested in the Einstein solution ( and not the way we get it) one could simply set them to zero by hand.
} 
are finite and has no UV divergences which usually appear as the effects of short term interactions. Nevertheless, if one subtracts the four dimensional Gauss-Bonnet action from the conformal action, as long as the entanglement entropy is concerned, the resultant action has the same physical content as that of the Einstein gravity.

The paper is organized as follows. In the next section we will study holographic entanglement entropy for black hole solutions in the conformal gravity in four dimensions where we observe that the resultant entanglement entropy is finite. Moreover for the Einstein solution this finite part is exactly the same as that one finds in the Einstein gravity using the minimal surface. In section three in order to explore our observation of the section two, we will study the entanglement entropy for the AdS plane wave solution of the model. The last section is devoted to discussions.

\section{Entanglement entropy for black brane solutions}

In this section we study holographic entanglement entropy for a black brane solution in the four dimensional conformal gravity. We will consider the cases where the entangling region is either a strip or a disk.

\subsection{Strip entangling region}

In this subsection we calculate entanglement entropy for an entangling region in the shape of a strip with the width of $\ell$. To do so, setting $\sum_{i=1}^{2} d x_{i}^{2}=d x^{2}+d y^{2}$ in the equation (1.4) the entangling region may be given by

$$
-\frac{\ell}{2} \leq x \leq \frac{\ell}{2}, \quad 0 \leq y \leq L_{y}, \quad t=\text { fixed }
$$

Then the corresponding co-dimension two hypersurface in the bulk may be parametrized by $t=0$ and $x=f(r)$ whose induced metric becomes ${ }^{3}$

$$
d s^{2}=\frac{L^{2}}{r^{2}}\left[\left(\frac{1}{b}+f^{\prime 2}\right) d r^{2}+d y^{2}\right] .
$$

Moreover the two unit vectors normal to the co-dimension two hypersurface are

$$
\begin{array}{lll}
\Sigma_{1}: & t=0 & n_{1}=\frac{L \sqrt{b}}{r}(1,0,0,0) \\
\Sigma_{2}: & x-f(r)=0 & n_{2}=\frac{L}{r \sqrt{1+b f^{\prime 2}}}\left(0,-f^{\prime}, 1,0\right) .
\end{array}
$$

Following the equation (1.9) one can compute the extrinsic curvatures of the hypersurface. Indeed one gets

$$
\mathcal{K}_{\mu \nu}^{(1)}=0, \quad \mathcal{K}_{\mu \nu}^{(2)}=\left(\begin{array}{cccc}
0 & 0 & 0 & 0 \\
0 & b^{-1} A & f^{\prime} A & \\
0 & f^{\prime} A & b f^{\prime 2} A & 0 \\
0 & 0 & 0 & B
\end{array}\right),
$$

\footnotetext{
${ }^{3}$ Through out this paper we will be dealing with three functions $f(r), b(r)$ and $k(r)$ which are functions of $r$. But in order to simplify the expressions we will drop their explicit dependence on $r$ and will write them as $f, b$ and $k$, respectively.
} 
where

$$
B=\frac{b L f^{\prime}}{r^{2} \sqrt{1+b f^{\prime 2}}}, \quad A=\frac{L\left[\left(2 b-r b^{\prime}\right) f^{\prime}+2 b^{2} f^{\prime 3}-2 b r f^{\prime \prime}\right]}{2 r^{2}\left(1+b f^{\prime 2}\right)^{5 / 2}} .
$$

Using the expression for the extrinsic curvatures and the normal vectors one can compute the entropy functional (1.8). In fact in the present case one finds

$$
S_{A}=-\frac{\kappa L_{y}}{4} \int d r\left[\frac{\left(b^{\prime} f^{\prime}+2 b f^{\prime \prime}\right)^{2}}{4 \sqrt{b}\left(1+b f^{\prime 2}\right)^{5 / 2}}+\frac{\left(2 b f^{\prime 2}-1\right) b^{\prime \prime}}{3 \sqrt{b} \sqrt{1+b f^{\prime 2}}}\right] .
$$

Now the aim is to minimize this entropy functional to find the profile of the hypersurface parametrized by $f$ with conditions that $f$ is a smooth differentiable function and $f(0)=\frac{\ell}{2}$. Before going into details of minimizing procedure, we would like to make a comment on the form of the above entropy functional.

Note that neither $L$ nor the radial coordinate $r$ appeared explicitly in the final form of the entropy functional. This observation together with the fact that both $b$ and $f$ are smooth differentiable functions, leads to an interesting prediction on the form of the entanglement entropy in this case. Namely, since the integrand does not diverge at $r=0$, the resultant entanglement entropy does not have UV divergent terms. This is unlike the area formula in the Einstein gravity where the integrand has the following typical divergent form

$$
A \sim \int d r \frac{\sqrt{1+b f^{\prime 2}}}{r^{d-1} \sqrt{b}} \quad \stackrel{r \rightarrow 0}{\longrightarrow} \quad A \sim \int_{\epsilon} \frac{d r}{r^{d-1}},
$$

where $\epsilon$ is a UV cut off. Therefore one may wonder how the conformal gravity would produce the Einstein gravity's results once the Neumann boundary condition on the metric is imposed. This is, indeed, the aim of this subsection to address this question.

In order to minimize the above entropy functional one may proceed with the well known procedure in the literature. Namely one may consider the entropy functional as a one dimensional action whose Lagrangian is defined by $S_{A}=\int d r \mathcal{L}$. Therefore, in the present case, the corresponding equation of motion is

$$
\frac{\partial^{2}}{\partial r^{2}}\left(\frac{\delta \mathcal{L}}{\delta f^{\prime \prime}}\right)-\frac{\partial}{\partial r}\left(\frac{\delta \mathcal{L}}{\delta f^{\prime}}\right)+\frac{\delta \mathcal{L}}{\delta f}=0 .
$$

Since the entropy functional (2.6) is independent of $f$, one gets a conservation law which might be solved to find $f$. We note, however, that in general it is difficult to solve the resultant equation. Of course for pure AdS solution where $b=1$ there is an exact solution as follows

$$
f^{\prime}(r)=\frac{r^{2}}{\sqrt{r_{t}^{4}-r^{4}}}
$$

where $r_{t}$ is the turning point where $f^{\prime}(r) \rightarrow \infty$.

Then, one may expand the equation around the AdS solution for small deformations of parameters $m$ and $a$. It is, however, important to note that since the equation of motion we get for $f^{\prime}(r)$ is a third order differential equation, in general it has three free parameters 
which should be fixed by proper boundary conditions. The corresponding condition we will impose are finiteness and reality conditions on $\ell$. More precisely at leading order one finds

$$
f^{\prime}(r)=\frac{r^{2}}{\sqrt{r_{t}^{4}-r^{4}}}\left[1+g(r)+\mathcal{O}\left(m^{2}, a^{2}, m a\right)\right]
$$

with

$$
\begin{aligned}
g(r)= & \frac{3 m r^{5}\left(r^{4}-r_{t}^{4}\right)+a r^{3}\left(r^{4}-2 r_{t}^{4}\right)-\sqrt{a m}\left(3 r^{8}-4 r^{4} r_{t}^{4}+r_{t}^{8}\right)}{6 r^{2}\left(r^{4}-r_{t}^{4}\right)}+\frac{c_{1}}{r\left(r^{4}-r_{t}^{4}\right)} \\
& +\frac{c_{2}}{2\left(r^{4}-r_{t}^{4}\right)}+c_{3}\left(\frac{1}{12 r^{2} r_{t}^{4} \sqrt{r_{t}^{4}-r^{4}}}+\frac{E\left(\sin ^{-1} \frac{r}{r_{t}},-1\right)-F\left(\sin ^{-1} \frac{r}{r_{t}},-1\right)}{6 r r_{t}^{3}\left(r_{t}^{4}-r^{4}\right)}\right) .
\end{aligned}
$$

where $E$ and $F$ are Elliptic functions. Using the fact that $\frac{\ell}{2}=\int_{0}^{r_{t}} d r f^{\prime}(r)$ one can find a relation between the width of the entangling region and the turning point. Of course the resultant width should be real and finite. Indeed, requiring these conditions, one finds

$$
c_{3}=0, \quad c_{2}=\frac{a}{3} r_{t}^{5}-\frac{2 c_{1}}{r_{t}} .
$$

Moreover in the limit of $\ell \rightarrow 0$, the turning point must approach zero. This condition would also require to set $c_{1}=0$. Therefore we arrive at ${ }^{4}$

$$
\begin{aligned}
f^{\prime}(r)=\frac{r^{2}}{\sqrt{r_{t}^{4}-r^{4}}\left[1+\frac{1}{2} m r^{3}+\right.} & \frac{a\left(r^{5}-2 r r_{t}^{4}+r_{t}^{5}\right)}{6\left(r^{4}-r_{t}^{4}\right)} \\
& \left.+\frac{\sqrt{a m}\left(r_{t}^{4}-3 r^{4}\right)}{6 r^{2}}+\mathcal{O}\left(m^{2}, a^{2}, m a\right)\right] .
\end{aligned}
$$

As a result, at leading order one gets

$$
\frac{\ell}{2}=\frac{\sqrt{\pi} \Gamma\left(\frac{3}{4}\right)}{\Gamma\left(\frac{1}{4}\right)} r_{t}+\frac{m \pi}{16} r_{t}^{4}+\frac{\sqrt{\pi} \Gamma\left(\frac{3}{4}\right)}{12 \Gamma\left(\frac{1}{4}\right)} a r_{t}^{2}+\mathcal{O}\left(m^{2}, a^{2}, m a\right),
$$

which can be inverted to find the turning point as a function of the width of the entangling region

$$
r_{t}=\frac{\Gamma\left(\frac{1}{4}\right)}{2 \sqrt{\pi} \Gamma\left(\frac{3}{4}\right)} \ell-\frac{\Gamma\left(\frac{1}{4}\right)^{5}}{256 \pi^{3 / 2} \Gamma\left(\frac{3}{4}\right)^{5}} m \ell^{4}-\frac{\Gamma\left(\frac{1}{4}\right)^{2}}{48 \pi \Gamma\left(\frac{3}{4}\right)^{2}} a \ell^{2}+\mathcal{O}\left(m^{2}, a^{2}, m a\right) .
$$

On the other hand plugging the profile (2.13) into the entropy functional one arrives at

$$
S_{E E}=-\frac{\kappa L_{y}}{4}\left[\frac{4 \sqrt{\pi} \Gamma\left(\frac{3}{4}\right)}{\Gamma\left(\frac{1}{4}\right)} \frac{1}{r_{t}}-\frac{m \pi}{2} r_{t}^{2}-\frac{\sqrt{\pi} \Gamma\left(\frac{3}{4}\right)}{3 \Gamma\left(\frac{1}{4}\right)} a\right]+\mathcal{O}\left(m^{2}, a^{2}, m a\right)
$$

\footnotetext{
${ }^{4}$ Note that in each order one needs to impose the reality and finiteness conditions on $\ell$.
} 
which, by making use of the equation (2.15), can be recast to the following form

$$
S_{E E}=\kappa L_{y}\left[-\frac{2 \pi \Gamma\left(\frac{3}{4}\right)^{2}}{\Gamma\left(\frac{1}{4}\right)^{2}} \frac{1}{\ell}+\frac{\Gamma\left(\frac{1}{4}\right)^{2}}{64 \Gamma\left(\frac{3}{4}\right)^{2}} m \ell^{2}\right]+\mathcal{O}\left(m^{2}, a^{2}, m a\right) .
$$

This is, indeed, our final result for the holographic entanglement entropy for a black hole solution in the conformal gravity.

An interesting feature of the resultant entanglement entropy is that it does not contain UV divergent terms, as we had already anticipated. Moreover those finite terms which are independent of $a$, up to an overall factor, are exactly the same as that in the Einstein gravity [19]. Therefore setting $a=0$, the entanglement entropy reduces to that of Einstein gravity which can be obtained from the minimal area.

It is worth noting that although the entanglement entropy by definition is a positive quantity, its finite term could be negative [19]. Therefore if with any procedure one removes the UV divergences of the theory, the resultant entanglement entropy could be negative. Having found a finite negative entanglement entropy for the conformal gravity, it would mean that the corresponding theory is intrinsically regularized. In what follows we will see this is, indeed, the case and moreover we show how the divergences could be detached and removed.

In fact the finiteness of the entanglement entropy may be understood from the fact that the four dimensional conformal gravity could be considered as a regularized four dimensional Einstein gravity [4]. So that all the divergences in the theory should have been removed. On the other hand following the results of [3] one would also expect that setting $a=0$ where the solution becomes a solution of Einstein gravity, the content of the model should also reduce to the Einstein gravity.

To explore these points better, note that the action of the four dimensional conformal gravity may be decomposed as follows

$$
\begin{aligned}
S & =-\frac{\kappa}{32 \pi} \int d^{4} x \sqrt{-g}\left(R_{\mu \nu \rho \sigma} R^{\mu \nu \rho \sigma}-2 R_{\mu \nu} R^{\mu \nu}+\frac{1}{3} R^{2}\right) \\
& =-\frac{\kappa}{32 \pi} \mathrm{GB}_{4}-\frac{\kappa}{16 \pi} \int d^{4} x \sqrt{-g}\left(R_{\mu \nu} R^{\mu \nu}-\frac{1}{3} R^{2}\right),
\end{aligned}
$$

where $\mathrm{GB}_{4}$ is the four dimensional Gauss-Bonnet action which is a total derivative and does not contribute to the equations of motion. Note that since the Gauss-Bonnet term is topological, the whole dynamics must be encoded in the second term. Therefore we will call the second term as the "dynamical term". ${ }^{5}$ It is illustrative to compute the contributions of these two terms to the entanglement entropy, separately.

\footnotetext{
${ }^{5}$ Actually there are several ways to decompose the Weyl action into a Gauss-Bonnet term plus a dynamical term. We note, however, that the decomposition (2.18) is special in a sense that the coefficients of the Weyl action and the Gauss-Bonnet action are the same. In other words the coefficient of the Gauss-Bonnet action is one up to the factor of $\frac{\kappa}{32 \pi}$. This is exactly the proper factor needed to regularize the four dimensional Einstein gravity with the Gauss-Bonnet term [20] (see also [3, 4]). Therefore the above dynamical terms is unique. As we will see, this leads to an interesting result concerning the connection between four dimensional conformal and Einstein gravities.
} 
To proceed let us consider the following action.

$$
S^{\mathrm{dyn}}=-\frac{\kappa}{16 \pi} \int d^{4} x \sqrt{-g}\left(R_{\mu \nu} R^{\mu \nu}-\frac{1}{3} R^{2}\right) .
$$

It is obvious that the black brane solution (1.4) is also a solution of the equations of motion of the above action. Moreover, following [14] the holographic entanglement entropy of a dual field theory whose gravitational description is given by the black brane solution of the above action must be obtained from the following entropy functional

$$
S_{A}^{\mathrm{dyn}}=\frac{\kappa}{4} \int d^{2} \zeta \sqrt{h}\left[-\frac{2}{3} R+\left(R_{\mu \nu} n_{i}^{\mu} n_{i}^{\nu}-\frac{1}{2} \mathcal{K}^{i} \mathcal{K}_{i}\right)\right]
$$

For the entangling region (2.1) and its corresponding co-dimension two hypersurface in the bulk, the above entropy functional reads

$$
\begin{gathered}
S_{A}^{\mathrm{dyn}}=\frac{\kappa L_{y}}{8} \int d r\left[\frac{6\left(3 b-r b^{\prime}\right)-\left(1-2 b f^{\prime 2}\right)\left(6 b-r^{2} b^{\prime \prime}\right)}{3 \sqrt{b} r^{2} \sqrt{1+b f^{\prime 2}}}\right. \\
\left.-\frac{\left(\left(4 b-r b^{\prime}\right) f^{\prime}+4 b^{2} f^{\prime 3}-2 r b f^{\prime \prime}\right)^{2}}{4 \sqrt{b} r^{2}\left(1+b f^{\prime 2}\right)^{5 / 2}}\right] .
\end{gathered}
$$

It is then straightforward to minimize the above entropy functional to read the corresponding profile of the hypersurface in the bulk. In fact solving the obtained equation perturbatively for small $a$ and $m$, we reach to the same profile as that in the equation (2.13). It is then easy to compute the entanglement entropy for the equation (2.20) which at leading order is

$$
S_{E E}^{\mathrm{dyn}}=\kappa L_{y}\left[\frac{1}{\epsilon}-\frac{a}{6}-\frac{2 \pi \Gamma\left(\frac{3}{4}\right)^{2}}{\Gamma\left(\frac{1}{4}\right)^{2}} \frac{1}{\ell}+\frac{\Gamma\left(\frac{1}{4}\right)^{2}}{64 \Gamma\left(\frac{3}{4}\right)^{2}} m \ell^{2}+\ldots\right] .
$$

Going through the same procedure for the Gauss-Bonnet term, one arrives at

$$
S_{E E}^{\mathrm{GB}}=\kappa L_{y}\left[-\frac{1}{\epsilon}+\frac{a}{6}+\ldots\right]
$$

It is then clear that taking both contributions into account the divergent term will drop leading to a finite entanglement entropy in the equation (2.17). Besides, setting $a=0$ where the solution becomes a Schwarzschild black brane, the entanglement entropy evaluated just by the dynamical part, $S_{E E}^{\text {dyn }}$, reduces to the entanglement entropy of a strip in the Einstein gravity if one identifies $\kappa$ as $\kappa=\frac{L^{2}}{2 G}$, where $G$ is the four dimensional Newton constant. ${ }^{6}$ It is worth recalling that setting $a=0$ corresponds to imposing the Neumann boundary condition on the metric which in turns reduces the solution to that of Einstein gravity [3]. ${ }^{7}$

\footnotetext{
${ }^{6}$ If we had considered another decomposition rather than (2.18) the result of the Gauss-Bonnet term would have been changed by an overall factor. Therefore the finite terms of the entanglement entropy, for an Einstein solution, do not depend on the decomposition. Of course in this case the whole entanglement entropy obtained just from the dynamical part would not be the same as that in the Einstein gravity.

${ }^{7}$ To compare our normalization with that in [3] one has $\kappa=32 \pi c_{W}$. See equation (3.6) of the paper [3].
} 
Actually we can show even more. Indeed the equation of motion obtained from the minimization of the equation (2.21) can be solved exactly leading to the following closed form for the profile of the hypersurface in the bulk

$$
f^{\prime}(r)=\frac{r^{2}}{\sqrt{b\left(r_{t}^{4}-r^{4}\right)}}, \quad \text { with } b=1-m r^{3} .
$$

We recognize that this is exactly the same profile which minimizes the area,

$$
S_{A}^{\text {Ein }}=\frac{L^{2} L_{y}}{4 G} \int d r \frac{\sqrt{1+b f^{\prime 2}}}{r^{2} \sqrt{b}},
$$

that is the entropy functional for the Einstein gravity. Evaluating the entropy functional coming from the dynamical part and the area function on this profile leads to the following expressions for the holographic entanglement entropy in the conformal and Einstein gravities, respectively

$$
S_{E E}^{\mathrm{dyn}}=\kappa L_{y} \int_{\epsilon}^{r_{t}} d r \frac{r_{t}^{2}}{r^{2} \sqrt{b\left(r_{t}^{4}-r^{4}\right)}}, \quad S_{E E}^{\mathrm{Ein}}=\frac{L^{2} L_{y}}{2 G} \int_{\epsilon}^{r_{t}} d r \frac{r_{t}^{2}}{r^{2} \sqrt{b\left(r_{t}^{4}-r^{4}\right)}},
$$

which are the same upon the identification of $\kappa=\frac{L^{2}}{2 G}$.

Therefore it is fair to conclude that for an Einstein solution the holographic entanglement entropy evaluated by the dynamical part contains the same information as the holographic entanglement entropy evaluated by the Einstein gravity where one has a simple proposal based on a minimal surface. This conclusion may be understood as follows. In fact by calculating the entanglement entropy, we are actually measuring the entanglement between different degrees of freedom located on a given region. Therefore only dynamical modes would contribute. On the other hand for the four dimensional conformal gravity the dynamics of the modes are governed by the dynamical part of the action. The topological Gauss-Bonnet term plays just the role of a regulator which regularizes the results. ${ }^{8}$

In order to further explore this observation, in the following subsection we redo our calculations for the case where the entangling region is a disk.

\subsection{Disk entangling region}

In this subsection we continue our studies on the holographic entanglement entropy of the four dimensional conformal gravity for an entangling region in the shape of a disk. To proceed we reparametrize the black brane metric as follows

$$
d s^{2}=\frac{L^{2}}{r^{2}}\left(-b d t^{2}+\frac{d r^{2}}{b}+d \rho^{2}+\rho^{2} d \phi^{2}\right), \quad b=1-\frac{a}{3} r+\sqrt{a m} r^{2}-m r^{3} .
$$

Consider a disk on the boundary theory with the radius of $\ell$ given by $\rho \leq \ell$, then the corresponding co-dimension two hypersurface in the bulk may be parametrized by $t=$ $0, \rho=f(r)$. Therefore the induced metric on the hypersurface is

$$
d s^{2}=\frac{L^{2}}{r^{2}}\left[\left(\frac{1}{b}+f^{\prime 2}\right) d r^{2}+f^{2} d \phi^{2}\right] .
$$

\footnotetext{
${ }^{8}$ We note that the role of the Gauss-Bonnet term as a regulator for AdS gravity action in four dimensions was also discussed in [21].
} 
Moreover, two unit vectors normal to the hypersurface are also given by

$$
\begin{array}{lll}
\Sigma_{1}: & t=0 & n_{1}=\frac{L \sqrt{b}}{r}(1,0,0,0) \\
\Sigma_{2}: & \rho-f(r)=0 & n_{2}=\frac{L}{r \sqrt{1+b f^{\prime 2}}}\left(0,-f^{\prime}, 1,0\right) .
\end{array}
$$

It is then easy to compute the extrinsic curvatures of the hypersurface associated to these vectors. Actually the results are the same as what we have found for the strip in the previous section, except that in the present case one has

$$
B=\frac{f L\left(r+b f f^{\prime}\right)}{r^{2} \sqrt{1+b f^{\prime 2}}}
$$

In this case the entropy functional, (1.8), reads

$$
S_{A}=-\frac{\pi \kappa}{4} \int d r\left[\frac{\left(2+f b^{\prime} f^{\prime}+2 b f^{\prime 2}+2 b f f^{\prime \prime}\right)^{2}}{4 \sqrt{b} f\left(1+b f^{\prime 2}\right)^{5 / 2}}+\frac{\left(2 b f^{\prime 2}-1\right) f b^{\prime \prime}}{3 \sqrt{b} \sqrt{1+b f^{\prime 2}}}\right] .
$$

The entropy functional associated to the dynamical part of the action can also be computed leading to the following expression

$$
\begin{aligned}
S_{A}^{\mathrm{dyn}}=\frac{\pi \kappa}{4} \int d r\left[\frac{6\left(3 b-r b^{\prime}\right)-\left(1-2 b f^{\prime 2}\right)\left(6 b-r^{2} b^{\prime \prime}\right)}{3 \sqrt{b} f^{-1} r^{2} \sqrt{1+b f^{\prime 2}}}\right. \\
\left.-\frac{\left[2\left(r+2 b f f^{\prime}\right)\left(1+b f^{\prime 2}\right)-r f\left(b^{\prime} f^{\prime}+2 b f^{\prime \prime}\right)\right]^{2}}{4 \sqrt{b} f r^{2}\left(1+b f^{\prime 2}\right)^{5 / 2}}\right]
\end{aligned}
$$

Now one needs to minimize the entropy functional to find a differential equation for the profile $f$. Following our observation in the previous subsection, as long as Einstein solutions are concerned one could only minimize the entropy functional associated to the dynamical part of action to find the profile. Also the entropy can be obtained from this part. It is, however, important to note that even for Einstein solutions the profile we find by the minimization of the entropy functional associated to the dynamical part does not necessarily minimize the whole entropy functional. This means that the part of entropy functional which comes from the topological term, might have non-trivial effects on the solution of the profile. Nevertheless as long as the finite parts of the entanglement entropy are concerned both of them lead to the same results for Einstein solutions. In what follows we will consider the entropy functional of whole system where the effects of both GaussBonnet and dynamical parts are taken into account.

Since in the present case the entropy functional depends on $f$, one does not have a conservation law and therefore the equation of motion has to be solved directly. Of course in general it is difficult to solve the resultant equation of motion. We note, however, that when the background is an AdS solution where $b=1$, the corresponding equation of motion admits an exact solution as follows

$$
f(r)=\sqrt{r_{t}^{2}-r^{2}}
$$


which is exactly the same as that in the Einstein gravity. For the black brane solution, following our previous example, with proper boundary conditions one can find a perturbative expansion for the profile for small $a$ and $m$ as follows

$$
\begin{aligned}
f(r)=\sqrt{r_{t}^{2}-r^{2}}[1+ & m \frac{2 r_{t}^{5}-r^{3}\left(r_{t}^{2}+r^{2}\right)}{8\left(r_{t}^{2}-r^{2}\right)}-\frac{\sqrt{m a}\left(\left(r+r_{t}\right)\left(r^{2}+2 r_{t}^{2}\right)-r_{t}^{3} \tanh ^{-1} \frac{r}{r_{t}}\right)}{6\left(r+r_{t}\right)} \\
& \left.+a \frac{r^{2}+r r_{t}+4 r_{t}^{2}}{12\left(r+r_{t}\right)}\right]+\mathcal{O}\left(m^{2}, a^{2}, m a\right),
\end{aligned}
$$

so that

$$
\ell=r_{t}\left[1+\frac{m}{4} r_{t}^{3}-\frac{\sqrt{m a}}{3} r_{t}^{2}+\frac{a}{3} r_{t}\right]+\mathcal{O}\left(m^{2}, a^{2}, m a\right)
$$

which can be used to find the turning point as a function of the radius of the entangling region, $\ell$. It easy to see that in the present case at leading order one just need to set $r_{t}=\ell$.

On the other hand form the entropy functional (2.31) one gets

$$
S_{E E}=\frac{\pi \kappa}{8} m r_{t}^{3}+\mathcal{O}(m, a, \sqrt{m a}),
$$

so that

$$
S_{E E}=\frac{\pi \kappa}{8} m \ell^{3}+\mathcal{O}(m, a, \sqrt{m a}),
$$

which is finite, as expected. It is worth nothing that if one sets $m=0$ and $a=0$ where the solution reduces to pure AdS geometry, the finite part of the entanglement entropy vanishes. It is unlike the entanglement entropy for a disk in Einstein gravity where the finite part is a universal constant [22]. To explore this point better, it is illustrative to compute the contributions of the dynamical part and the Gauss-Bonnet term to the entanglement entropy separately. Indeed for the dynamical part one finds

$$
S_{E E}^{\mathrm{dyn}}=\pi \kappa\left[\frac{\ell}{\epsilon}-1+\frac{m \ell^{3}}{8}-\frac{5 a \ell}{12}+\frac{\sqrt{a m} \ell^{2}}{6}+\cdots\right]
$$

while the Gauss-Bonnet contribution to the entanglement entropy is

$$
S_{E E}^{\mathrm{GB}}=\pi \kappa\left[-\frac{\ell}{\epsilon}+1+\frac{5 a^{2} \ell}{12}-\frac{\sqrt{m a} \ell^{2}}{6}+\cdots\right]
$$

As one observes both dynamical part and Gauss-Bonnet term contribute to the universal part but with opposite signs. Therefore the universal part drops when both contributions are taken into account.

It is also clear that when one sets $a=0$ where the solution is a four dimensional Schwarzschild black brane of the Einstein gravity, the contribution of the dynamical part is exactly the same as the entanglement entropy obtained from minimal surface in the Einstein gravity if one identifies $\kappa=\frac{L^{2}}{2 G}$. The Gauss-Bonnet term plays the role of a regulator and its contribution removes the divergent term. 


\section{Entanglement entropy for wave solution}

In this section in order to further explore the connection between holographic entanglement entropy in the four dimensional conformal gravity and that in the Einstein gravity we will study entanglement entropy for a field theory whose gravitational description is given by the four dimensional conformal gravity on an AdS plane wave. It is another non-trivial example where the important role of the dynamical part of the action may be also seen.

As we have already mentioned, the equations of motion of the conformal gravity admit an AdS plane wave solution as follows

$$
d s^{2}=\frac{L^{2}}{r^{2}}\left[d r^{2}-2 d x_{+} d x_{-}+k(r) d x_{+}^{2}+d y^{2}\right], \quad k(r)=c_{0}+c_{1} r+c_{2} r^{2}+c_{3} r^{3} .
$$

The constant $c_{0}$ can be set to zero by a shift. When $c_{1}$ and $c_{2}$ are non-zero this is only a solution of conformal gravity. Since in what follows we are interested in the Einstein solution, we set $c_{1}=c_{2}=0$, so that $k=m r^{3}$.

Let us consider a strip in the dual theory whose width is extended along $y$ direction. More precisely one has

$$
\left(x_{-}, x_{+}\right)=(-z, z), \quad \frac{\ell}{2} \leq y \leq \frac{\ell}{2}, \quad t=\text { fixed }
$$

Then the co-dimension two hypersurface in the bulk may be given by $y=f(r)$. Therefore two unit vectors normal to the hypersurface are

$$
\begin{array}{ll}
\Sigma_{1}: x_{+}+x_{-}=0 & n_{1}=\frac{L}{r \sqrt{2+k}}(0,1,1,0) \\
\Sigma_{2}: y-f(r)=0 & n_{2}=\frac{L}{r \sqrt{1+f^{\prime 2}}}\left(-f^{\prime}, 0,0,1\right) .
\end{array}
$$

The induced metric on the co-dimension two hypersurface in the bulk becomes

$$
d s^{2}=\frac{L^{2}}{r^{2}}\left[\left(1+f^{\prime 2}\right) d r^{2}+(2+k) d z^{2}\right] .
$$

Entanglement entropy for a field theory whose dual is the Einstein gravity on the above wave solution has been studied in [23]. In this case, being Einstein gravity, one only needs to consider the area of the hypersurface

$$
S_{A}^{\text {Ein }}=\frac{L^{2} L_{+}}{4 \sqrt{2} G} \int d r \frac{\sqrt{(2+k)\left(1+f^{\prime 2}\right)}}{r^{2}} .
$$

It is easy then to minimize this area to find the profile of the hypersurface in the bulk which is

$$
f^{\prime}(r)=\frac{c r^{2}}{\sqrt{2+k-c^{2} r^{4}}} .
$$

where $c=\sqrt{2+k\left(r_{t}\right)} / r_{t}^{2}$, with $r_{t}$ being the turning point, is a constant of motion. Plugging the profile in the area, one can read the entanglement entropy

$$
S_{E E}^{\operatorname{Ein}}=\frac{L^{2} L_{+}}{2 \sqrt{2} G} \int_{\epsilon}^{r_{t}} d r \frac{(2+k)}{r^{2} \sqrt{2+k-c^{2} r^{4}}} .
$$


Now let us consider the case of conformal gravity. Following our results in the previous section for an Einstein solution the physical information is encoded in entropy functional associated to the dynamical part of the action (2.20). For our entangling region the entropy functional associated to the dynamical part reads

$$
\begin{aligned}
& S_{A}^{\mathrm{dyn}}=\frac{\kappa L_{+}}{8 \sqrt{2}} \int d r\left[\frac{\sqrt{1+f^{\prime 2}}\left(8+4 k-2 r k^{\prime}+r^{2} k^{\prime \prime}\right)}{r^{2} \sqrt{2+k}}\right. \\
&\left.-\frac{\left[f^{\prime}\left(1+f^{\prime 2}\right)\left(-8-4 k+r k^{\prime}\right)+2 r(2+k) f^{\prime \prime}\right]^{2}}{4 r^{2}(2+k)^{3 / 2}\left(1+f^{\prime 2}\right)^{5 / 2}}\right] .
\end{aligned}
$$

The profile of the hypersurface is obtained by minimizing this entropy functional. Although the equation of motion is lengthy, one can easily verify that the profile (3.6) is still a solution of the corresponding equation. Therefore entanglement entropy can be calculated by plugging the profile (3.6) into the above entropy functional. Doing so, one arrives at

$$
S_{E E}^{\mathrm{dyn}}=\frac{\kappa L_{+}}{\sqrt{2}} \int_{\epsilon}^{r_{t}} \frac{(2+k)}{r^{2} \sqrt{2+k-c^{2} r^{4}}},
$$

which is exactly the same as that in the equation (3.7) with a proper identification of $\kappa$ as we have done in the previous section. Therefore, taking the results of [3] into account it is natural to consider the Gauss-Bonnet term as just a regulator which removes the divergency of the resultant entanglement entropy. Of course in order to explore the role of Gauss-Bonnet term it is important to minimize the whole entropy functional when both the dynamical part and Gauss-Bonnet term are taken into account.

For the entangling region we are considering in this section, the entropy functional (1.8) reads $^{9}$

$$
\begin{aligned}
S_{A}=-\frac{\kappa L_{+}}{8 \sqrt{2}} \int d r\left[\frac{k^{\prime 2}\left(-4+f^{\prime 2}\right)}{4(2+k)^{\frac{3}{2}} \sqrt{1+f^{\prime 2}}}\right. & \\
& \left.+\frac{(2+k) f^{\prime \prime 2}-k^{\prime} f^{\prime}\left(1+f^{\prime 2}\right) f^{\prime \prime}+\left(1-f^{\prime 2}\right)\left(1+f^{\prime 2}\right)^{2} k^{\prime \prime}}{\sqrt{2+k}\left(1+f^{\prime 2}\right)^{\frac{5}{2}}}\right] .
\end{aligned}
$$

It is then straightforward to minimize this entropy functional to get a differential equation for the profile $f$ which indeed leads to the same profile as (3.6). Therefore it is straightforward to redo our computations for the whole entropy functional to explore the role of Gauss-Bonnet term.

By performing an integration from the equation (3.6) one may find the width of the strip as a function of the turning point. Indeed for small $m$ at leading order one finds

$$
f^{\prime}(r)=\frac{r^{2}}{\sqrt{r_{t}^{4}-r^{4}}}\left[1+\frac{m r_{t}^{4}\left(r^{3}-r_{t}^{3}\right)}{4\left(r^{4}-r_{t}^{4}\right)}\right]+\cdots,
$$

\footnotetext{
${ }^{9}$ There was a sign mistake in the equation (3.10) in the first version of the paper. This was due to our Mathematica code. Indeed this equation which contains both the contributions of the dynamical and the Gauss-Bonnet parts to the entropy functional can be found with different methods. Actually we have checked this equation with different ways which eventually leads to find our mistake in our Mathematica code. We would like to thank the referee for pointing out our mistake and suggesting a way to check our computations.
} 
which can be integrated to get

$$
\frac{\ell}{2}=\frac{\sqrt{\pi} \Gamma\left(\frac{3}{4}\right)}{\Gamma\left(\frac{1}{4}\right)} r_{t}+\frac{m r_{t}^{4}}{16}\left(\pi-\frac{2 \sqrt{\pi} \Gamma\left(\frac{3}{4}\right)}{\Gamma\left(\frac{1}{4}\right)}\right)+\cdots .
$$

This can be inverted to find the turning point as a function of the width of strip as follows

$$
r_{t}=\frac{\Gamma\left(\frac{1}{4}\right)}{2 \sqrt{\pi} \Gamma\left(\frac{3}{4}\right)} \ell+\frac{\Gamma\left(\frac{1}{4}\right)^{4}\left(-\sqrt{\pi} \Gamma\left(\frac{1}{4}\right)+2 \Gamma\left(\frac{3}{4}\right)\right)}{256 \pi^{2} \Gamma\left(\frac{3}{4}\right)^{5}} m \ell^{4}+\cdots .
$$

It is then easy to compute the contributions of the dynamical part and the Gauss-Bonnet term to the entanglement entropy which are

$$
\begin{aligned}
S_{E E}^{G B} & =-\kappa L_{+} \frac{1}{\epsilon}+\cdots, \\
S_{E E}^{d y n .} & =\kappa L_{+}\left[\frac{1}{\epsilon}-\frac{2 \pi \Gamma\left(\frac{3}{4}\right)^{2}}{\Gamma\left(\frac{1}{4}\right)^{2}} \frac{1}{\ell}+\frac{\Gamma\left(\frac{1}{4}\right)^{2}}{64 \Gamma\left(\frac{3}{4}\right)^{2}} m \ell^{2}\right]+\cdots,
\end{aligned}
$$

that have the expected forms. Namely the Gauss-Bonnet term plays the role of a regulator and the dynamical part reduces to that of Einstein gravity.

\section{Discussions}

In this paper we have studied entanglement entropy of a quantum field theory whose gravitational description is provided by a four dimensional conformal gravity. Since conformal gravities, typically, contain higher derivative terms, the simple holographic description of the entanglement entropy based on the minimal area is not applicable. Therefore in order to compute the entanglement entropy we have used the prescription introduced in [14].

By making use of this method we have computed holographic entanglement entropy for a conformal gravity in four dimensions where we have found that the resultant entanglement entropy, unlike the known examples in the literature, is finite and has no UV divergences. The finiteness of the entanglement entropy may be understood from the fact that the Weyl action in four dimensions is equal to regularized on shell action of the Einstein gravity when all (classical) counter-terms in the bulk are taken into account. Therefore, using the holographic renormalization, the UV divergences of the dual field theory which in turns correspond to the infinite volume limit in the bulk must be absent. Note that the finiteness of the entanglement entropy occurs both for Einstein and non-Einstein solutions. Of course, this is the case due to the fact that the finiteness is related to the regularization of the volume in the bulk and moreover, since both solutions have the same asymptotic form, the volume regularization is the same for both of them.

Another interesting observation we have made is as follows. Actually when one computes the holographic entanglement entropy for an Einstein solution of the conformal gravity the obtained entanglement entropy is exactly the same as the finite part of the entanglement entropy in the Einstein gravity. This might also be understood from the fact that the four dimensional conformal gravity with certain boundary condition, has the same physical content as that of the Einstein gravity in four dimensions. 
To explore this point better we note that the action of the four dimensional conformal gravity can be decomposed into two parts. The first part is just the four dimensional GaussBonnet term which is topological and dose not contribute to the equations of motion. The other part which we have called it "dynamical part" governs the dynamics of the system. As long as the equations of motion and their solutions are concerned the dynamical part plays the main role. Whereas, if we would like to compute the thermal entropy or the energy of the solution the contribution of the Gauss-Bonnet term is essential as well. Indeed in order to get the first law of the black hole thermodynamics it is important to consider the contribution of the Gauss-Bonnet term too.

On the other hand, in this paper by explicit examples, we have shown that for an Einstein solution the contribution of the dynamical part of the action to the entanglement entropy is exactly the same as that in the Einstein gravity where the entanglement entropy is computed by minimizing the area of a co-dimension two hypersurface in the bulk. It is quite a non-trivial result, taking into account that in conformal gravity the entropy functional is not the area and indeed has rather a complicated expression. Note also that the obtained profile of the hypersurface in the bulk minimizes both the area and the entropy functional corresponding to the dynamical part of the conformal gravity. Although we have shown this connection for certain entangling regions, it is natural to conjecture that this is, indeed, the case for an arbitrary entangling region.

Moreover, as we have already mentioned the entanglement entropy obtained from total action where the contribution of the Gauss-Bonnet is also taken into account is finite. This fact together with the above observation indicates that the Gauss-Bonnet term plays the role of a regulator which makes the theory finite. It is then natural to imagine that the field theory dual to the four dimensional conformal gravity, whatever it is, is finite.

It is important to note that the above conclusion makes sense for those theories whose gravitational descriptions are provided by conformal gravity on asymptotically locally AdS solutions where the results of [4] is applied. Indeed, if one drops the assumption of being "asymptotically hyperbolic" for the metric the results of [4] fails to hold and therefore there is no relation between Weyl action and regularized four dimensional on shell Einstein action. Therefore the corresponding entanglement entropy is not finite. One can verify this statement with an explicit example.

Actually the conformal gravity in four dimensions has the following $z=4$ Lifshitz black hole solution [10]

$$
d s^{2}=\frac{L^{2}}{r^{2}}\left[-\frac{b}{r^{6}} d t^{2}+\frac{d r^{2}}{b}+\sum_{i=1}^{2} d x_{i}^{2}\right], \quad b=1+c_{1} r^{2}+\frac{c_{1}^{2}}{3} r^{4}+c_{2} r^{6}
$$

which is not asymptotically AdS solution. It is then easy to compute the holographic entanglement entropy for this solution. Indeed setting $b=1$ (for simplicity) the entropy functional (1.8) for the strip (2.1) reads

$$
S_{A}=\frac{\kappa L_{y}}{8} \int d r\left[\frac{8-8 f^{\prime 4}\left(3+2 f^{\prime 2}\right)-r^{2} f^{\prime \prime 2}}{r^{2}\left(1+f^{\prime 2}\right)^{5 / 2}}\right]
$$


which leads to the following UV divergent term in the entanglement entropy

$$
S_{E E}=\kappa L_{y} \frac{1}{\epsilon}+\cdots
$$

One observes that, even though, the Gauss-Bonnet term is also taken into account the result is not UV finite. More precisely doing the same for dynamical and Gauss-Bonnet parts one finds

$$
S_{E E}^{\mathrm{dyn}}=\frac{3 \kappa L_{y}}{2} \frac{1}{\epsilon}+\cdots \quad S_{E E}^{\mathrm{GB}}=-\frac{\kappa L_{y}}{2} \frac{1}{\epsilon}+\cdots
$$

As a side comment, note that from our computations one may calculate the variation of entanglement entropy when the system changes from a ground state to an excited state. Besides, since in all cases we have considered that the metric is asymptotically AdS, it is possible to compute the expectation value of the energy momentum tensor of the dual field theory using holographic renormalization. Therefore it is possible to verify whether the resultant variation of energy and entanglement entropy satisfy the first law of the entanglement thermodynamics [19, 24-26]. Actually one finds that for an Einstein solution, the variation of the entanglement entropy and energy satisfy the first law of the entanglement thermodynamics with the same entanglement temperature as that in the Einstein gravity. Indeed, unlike the first law of black hole thermodynamics, the Gauss-bonnet term has no contribution.

We note, however, that for non-Einstein solutions (for example when $a \neq 0$ ) the GaussBonnet term does contribute. For example if one looks at the expression of $S_{E E}^{\text {dyn }}$ for the sphere, (2.38) and try to read the first law from this paper, due to $\sqrt{m a}$-term one finds an extra term in the first law of the entanglement thermodynamics proportional to $\sqrt{a}$. On the other hand if one reads the first law from the total entanglement entropy due to the contribution of the Gauss-Bonnet the extra term drops from the equation, leading to the same relation as that of the Einstein gravity.

\section{Acknowledgments}

We would like to thank A. Naseh for a discussion. We also acknowledge the use of M. Headrick's excellent Mathematica package "diffgeo". We would like to thank him for his generosity. We would also like to thank the referee for his/her useful comments, specially his/her comment on a mistake in the equation (3.10).

Open Access. This article is distributed under the terms of the Creative Commons Attribution License (CC-BY 4.0), which permits any use, distribution and reproduction in any medium, provided the original author(s) and source are credited.

\section{References}

[1] K. Stelle, Renormalization of higher derivative quantum gravity, Phys. Rev. D 16 (1977) 953 [INSPIRE]. 
[2] S.L. Adler, Einstein gravity as a symmetry breaking effect in quantum field theory, Rev. Mod. Phys. 54 (1982) 729 [Erratum ibid. 55 (1983) 837] [INSPIRE].

[3] J. Maldacena, Einstein gravity from conformal gravity, arXiv:1105.5632 [INSPIRE].

[4] M.T. Anderson, $L^{2}$ curvature and volume renormalization of AHE metrics on 4-manifolds, Math. Res. Lett. 8 (2001) 171 [math/0011051].

[5] R.J. Riegert, Birkhoff's theorem in conformal gravity, Phys. Rev. Lett. 53 (1984) 315 [INSPIRE].

[6] D. Grumiller, Model for gravity at large distances, Phys. Rev. Lett. 105 (2010) 211303 [Erratum ibid. 106 (2011) 039901] [arXiv:1011.3625] [INSPIRE].

[7] E. Witten, Anti-de Sitter space, thermal phase transition and confinement in gauge theories, Adv. Theor. Math. Phys. 2 (1998) 505 [hep-th/9803131] [INSPIRE].

[8] M. Alishahiha and R. Fareghbal, D-dimensional log gravity, Phys. Rev. D 83 (2011) 084052 [arXiv:1101.5891] [INSPIRE].

[9] I. Gullu, M. Gurses, T.C. Sisman and B. Tekin, AdS waves as exact solutions to quadratic gravity, Phys. Rev. D 83 (2011) 084015 [arXiv:1102.1921] [INSPIRE].

[10] H. Lü, Y. Pang, C.N. Pope and J.F. Vazquez-Poritz, AdS and Lifshitz black holes in conformal and Einstein-Weyl gravities, Phys. Rev. D 86 (2012) 044011 [arXiv:1204.1062] [INSPIRE].

[11] D. Grumiller, M. Irakleidou, I. Lovrekovic and R. McNees, Conformal gravity holography in four dimensions, arXiv:1310.0819 [INSPIRE].

[12] A. Naseh, unpublished.

[13] S. Ryu and T. Takayanagi, Holographic derivation of entanglement entropy from AdS/CFT, Phys. Rev. Lett. 96 (2006) 181602 [hep-th/0603001] [INSPIRE].

[14] D.V. Fursaev, A. Patrushev and S.N. Solodukhin, Distributional geometry of squashed cones, arXiv: 1306.4000 [INSPIRE].

[15] A. Bhattacharyya, M. Sharma and A. Sinha, On generalized gravitational entropy, squashed cones and holography, JHEP 01 (2014) 021 [arXiv:1308.5748] [INSPIRE].

[16] M. Alishahiha, A.F. Astaneh and M.R.M. Mozaffar, Entanglement entropy for logarithmic conformal field theory, arXiv:1310.4294 [INSPIRE].

[17] X. Dong, Holographic entanglement entropy for general higher derivative gravity, JHEP 01 (2014) 044 [arXiv: 1310.5713] [INSPIRE].

[18] J. Camps, Generalized entropy and higher derivative Gravity, arXiv:1310.6659 [INSPIRE].

[19] J. Bhattacharya, M. Nozaki, T. Takayanagi and T. Ugajin, Thermodynamical property of entanglement entropy for excited states, Phys. Rev. Lett. 110 (2013) 091602 [arXiv: 1212.1164] [INSPIRE].

[20] O. Mišković and R. Olea, Topological regularization and self-duality in four-dimensional Anti-de Sitter gravity, Phys. Rev. D 79 (2009) 124020 [arXiv:0902.2082] [INSPIRE].

[21] R. Olea, Mass, angular momentum and thermodynamics in four-dimensional Kerr-AdS black holes, JHEP 06 (2005) 023 [hep-th/0504233] [INSPIRE].

[22] S. Ryu and T. Takayanagi, Aspects of holographic entanglement entropy, JHEP 08 (2006) 045 [hep-th/0605073] [INSPIRE]. 
[23] K. Narayan, T. Takayanagi and S.P. Trivedi, AdS plane waves and entanglement entropy, JHEP 04 (2013) 051 [arXiv: 1212.4328] [INSPIRE].

[24] D. Allahbakhshi, M. Alishahiha and A. Naseh, Entanglement thermodynamics, JHEP 08 (2013) 102 [arXiv:1305.2728] [INSPIRE].

[25] D.D. Blanco, H. Casini, L.-Y. Hung and R.C. Myers, Relative entropy and holography, JHEP 08 (2013) 060 [arXiv: 1305.3182] [INSPIRE].

[26] G. Wong, I. Klich, L.A. Pando Zayas and D. Vaman, Entanglement temperature and entanglement entropy of excited states, JHEP 12 (2013) 020 [arXiv: 1305.3291] [INSPIRE]. 